\title{
Small Molecule Inhibitors of CRM1
}

\author{
Bibiana I. Ferreira ${ }^{1,2,3}$, Bastien Cautain ${ }^{4,5}$, Inês Grenho ${ }^{1,2,3}$ and Wolfgang Link ${ }^{6 *}$ \\ ${ }^{1}$ Centre for Biomedical Research (CBMR), University of Algarve, Faro, Portugal, 2 Regenerative Medicine Program, \\ Department of Biomedical Sciences and Medicine, University of Algarve, Faro, Portugal, ${ }^{3}$ Algarve Biomedical Center (ABC), \\ University of Algarve, Faro, Portugal, ${ }^{4}$ Fundacion MEDINA Parque tecnológico ciencias de la salud, Granada, Spain, 5 Evotec \\ France, Toulouse, France, 6 Instituto de Investigaciones Biomédicas "Alberto Sols" (CSIC-UAM), Madrid, Spain
}

\section{OPEN ACCESS}

Edited by:

Patrícia Mendonça Rijo, Universidade Lusófona Research Center for Biosciences \& Health Technologies, Portugal

Reviewed by: Hsu-Wen Chao, Taipei Medical University, Taiwan Yuh Min Chook,

University of Texas Southwestern Medical Center, United States Célia Maria Cardona Faustino, University of Lisbon, Portugal

*Correspondence: Wolfgang Link walink@iib.uam.es

Specialty section: This article was submitted to Translational Pharmacology, a section of the journal Frontiers in Pharmacology

Received: 10 December 2019 Accepted: 20 April 2020 Published: 07 May 2020

Citation:

Ferreira BI, Cautain B, Grenho I and Link W (2020) Small Molecule Inhibitors of CRM1.

Front. Pharmacol. 11:625. doi: 10.3389/fphar.2020.00625
The transport through the nuclear pore complex is used by cancer cells to evade tumorsuppressive mechanisms. Several tumor-suppressors have been shown to be excluded from the cell nucleus in cancer cells by the nuclear export receptor CRM1 and abnormal expression of CRM1 is oncogenic. Inhibition of CRM1 has long been postulated as potential approach for the treatment of cancer and to overcome therapy resistance. Furthermore, the nuclear export of viral components mediated by the CRM1 is crucial in various stages of the viral lifecycle and assembly of many viruses from diverse families, including coronavirus. However, the first nuclear export inhibitors failed or never entered into clinical trials. More recently CRM1 reemerged as a cancer target and a successful proof of concept was achieved with the clinical approval of Selinexor. The chemical complexity of natural products is a promising perspective for the discovery of new nuclear export inhibitors with a favorable toxicity profile. Several screening campaigns have been performed and several natural product-based nuclear export inhibitors have been identified. With this review we give an overview over the role of CRM1-mediated nuclear export in cancer and the effort made to identify and develop nuclear export inhibitors in particular from natural sources.

Keywords: CRM1, nuclear export, natural products (NP), leptomycin B, Selinexor, high content screening (HCS)

\section{INTRODUCTION}

Human diseases often involve alterations in the structure, localization, interactions, and as a consequence, the function of cellular proteins (Hung and Link, 2011). Normal cell physiology requires a tightly regulated, coordinated activity of thousands of proteins, that must be in the right place at the right time. Protein function depends on the subcellular localization as it determines access of the protein to binding partners and enzymes that catalyze post-translational modification and facilitates its contribution to functional networks. For example, transcription factors have to be in the cell nucleus in order to have access to the target gene promoters and to exert their transcriptional activity. With the exception of red blood cells, all eukaryotic cells contain a nucleus which is surrounded by a nuclear envelope made up of two lipid bilayer membranes. The nuclear envelope represents a greater physical barrier than the single lipid bilayer of the plasma membrane. The movement of molecules between the nucleoplasm and the cytoplasm is controlled by nuclear pore complexes (NPCs). The NPC is one of the largest cellular protein complexes and is responsible for the controlled passage of macromolecules into and out of the nucleus. The NPCs are permeable only to small molecules such as salts, nucleotides, small proteins. Proteins over $40 \mathrm{kDa}$ are required 
to be moved through the nuclear pore complex (NPCs) by soluble nuclear transport receptors (Cautain et al., 2015b). Proteins that enter and exit through the NPC usually contain specific transport signals namely a nuclear localization signal (NLS) or a nuclear export signal (NES). These sequences are recognized by soluble transport receptors of the karyopherin family.

\section{THE CRM1 EXPORT RECEPTOR}

The best studied export protein is chromosome region maintenance 1 (CRM1 also known as XPO1 or exportin 1) (Fornerod et al., 1997; Fukuda et al., 1997; Ossareh-Nazari et al., 1997; Kudo et al., 1998). CRM1 is expressed in all eukaryotic cells. CRM1 belongs to the karyopherin- $\beta$ family of transport receptors and mediates the nuclear exports of proteins that contain leucine-rich NESs (Fukuda et al., 1997). Over 200 proteins have been verified as cargoes of CRM1. Ran-GTP binds to CRM1 in the nucleus causing an increased affinity to the NES containing cargo protein and a complex between Ran, CRM1 and the cargo forms. This complex is exported through the NPC into the cytoplasm where Ran-GTP is converted into Ran-GDP and the complex dissociates. Human CRM1 consists of 1071 amino-acid residues and contains several functional regions (Figure 1). CRM1 is made up of 21 tandemly repeated, 37-47 amino acid long modules called HEAT protein domains (Dong et al., 2009a; Dong et al., 2009b; Monecke et al., 2009). Each repeat forms a hairpin of two helices called A and B helices building a ring-shaped structure. While the outer surface of the ring comprise the $\mathrm{A}$ helices, the $\mathrm{B}$ helices form the inner surface (Güttler et al., 2010; Koyama and Matsuura, 2010; Dian et al., 2013; Monecke et al., 2013; Saito and Matsuura, 2013). The NES binding cleft is formed by HEAT repeats 11 and 12 at the outer surface of CRM1. The N-terminal CRIME domain shares sequence homology with importin- $\beta$ and is involved in binding to RanGTP. The acidic loop within HEAT-repeat 9 also contributes to RanGTP binding and is thought to inhibit the interaction with NES in the absence of RanGTP. While the central part of CRM1 is involved the interaction with NES, the C-terminal end of CRM1 is thought to modulate its affinity to NES (Dong et al., 2009a; Dian et al., 2013).

\section{The Role of CRM1 in Cancer}

CRM1 is responsible for the nuclear export of a large number of tumor suppressor and oncogenic proteins including retinoblastoma, APC, FOXO proteins, INI1/hSNF5, galectin-3, Bok, NPM1, RASSF2, Merlin, p53, p21CIP, p27KIP1, N-WASP/ FAK, estradiol receptor, Tob, BRAC1, BCR-ABL and eIF4E. Importantly, many of these proteins were found to be mislocalized in cancer cells (Hill et al., 2014). RNAi-mediated silencing of the CRM1 induced nuclear retention of p53 and cell death in cervical cancer cell lines (Santiago et al., 2013). Somatic mutations in CRM1 have been identified in chronic lymphocytic leukemia (Puente et al., 2011) and in other hematological malignancies (Sendino et al., 2018). The great majority of these mutations affect a single amino acid at position 571 . The E571K mutation localizes near the NES-binding site and substitutes a glutamic acid with a lysine. This variation replaces a negatively charged residue with a positively charged amino acid and might lead to an increased affinity for NES (García-Santisteban et al., 2016).

The expression of CRM1 is increased in a broad variety of cancer types including cervical (van der Watt et al., 2009), ovarian (Noske et al., 2008), kidney (Inoue et al., 2013), lung (Gao et al., 2015) and gastric cancers (Zhou et al., 2013), as well as in glioma (Shen et al., 2009), osteosarcoma (Yao et al., 2009), esophageal carcinoma (Van Der Watt et al., 2014), hepatocellular carcinoma (Zheng et al., 2014), multiple myeloma (Schmidt et al., 2013), acute myeloid leukemia (Kojima et al., 2013), chronic myeloid/lymphoid leukemia (Lapalombella et al., 2012), mantle cell lymphoma (Yoshimura et al., 2014) and plasma cell leukemia (Tai et al., 2014). In line with these observations, high level of CRM1 expression is correlated with tumor size, the presence of distant metastasis and poor prognosis in many cancer types. Therefore, CRM1 expression might have the potential to predict clinical outcome for several human tumor types. Importantly, CRM1 also plays an important role in drug resistance (Turner and Sullivan, 2008; Turner et al., 2012). Several different nuclear export inhibitors (NEIs) have been shown to sensitize drug-resistant cancer cells to anti-cancer drugs. These data suggest that interfering with the nuclear export

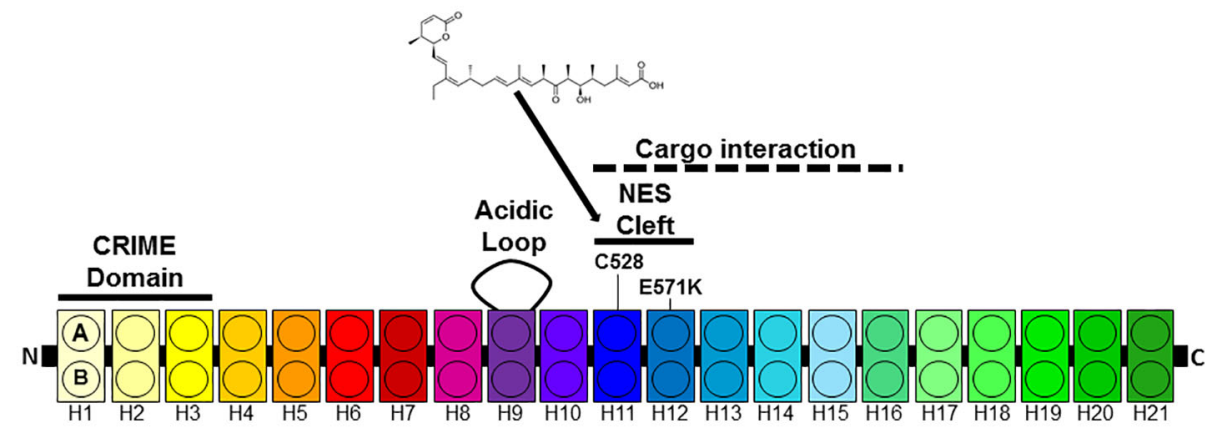

FIGURE 1 | Architecture of CRM1. CRM1 consists of 21 HEAT repeats each composed of two anti-parallel helices (A, B) connected by a linker loop. The Nterminal CRIME domain and the acidic loop contribute to RanGTP binding. The A helices of HEAT tandem 11 and 12 form the NES cleft. HEAT repeats $11-16$ are involved in cargo e.g. Snurportin1 (Spn1) binding. 
of tumor suppressor proteins or cell cycle inhibitors might contribute to overcome therapy resistance.

\section{Binding of CRM1 to Leptomycin B}

The progress made in understanding CRM1-mediated nuclear export is greatly based on the identification of Leptomycin B (LMB) as a CRM1 inhibitor (Kudo et al., 1998). LMB is natural product polyketide isolated from Streptomyces and has been originally discovered as a potent antifungal compound (Hamamoto et al., 1983). LMB contains two conjugated dienes, an $\alpha, \beta$-unsaturated $\delta$-lactone, a $\beta$-hydroxy-ketone moiety, and a terminal carboxylate (Figure 2A). Its molecular weight is 540Da. LMB binds covalently to a cysteine residue in CRM1 in HEAT repeat 11 of CRM1 (Cys-528 in the human CRM1) which is located in the NES-binding groove by a Michael-type addition reaction via its $\alpha, \beta$-unsaturated $\delta$-lactone moiety (Kudo et al., 1999a). As LMB modifies a cysteine residue in CRM1 critical for NES-cargo binding, it inhibits the formation of the NES-CRM1RanGTP complex and thereby the export of the cargo protein to the cytoplasm. Surprisingly, CRM1 acts as an enzyme hydrolyzing the lactone of LMB and thereby optimizing the LMB-CRM1 interaction. CRM1-induced modification of LMB leads to the irreversibility of the conjugation (Sun et al., 2013). LMB showed promising anti-cancer activity in preclinical experiments, but failed in clinical trial due to its systemic toxicity (Newlands et al., 1996). The dose limiting toxicity associated with LMB is thought to be due to a permanent block of nuclear export of essential macromolecules.

A

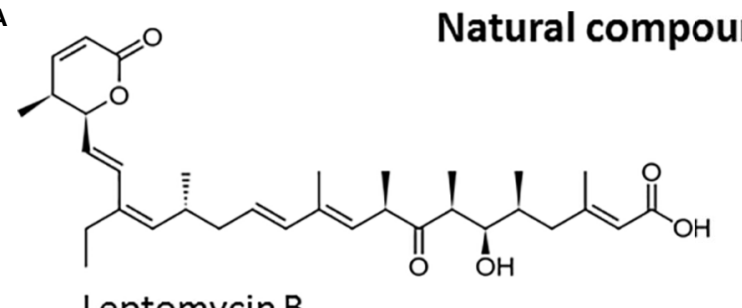

Leptomycin B

Source: Bacteria

MoA: Binding CRM1 C528

Acetoxychavicol acetate

Source: Plant

$\stackrel{\mathrm{OH}}{\mathrm{H}}$ MoA: Binding CRM1 C528

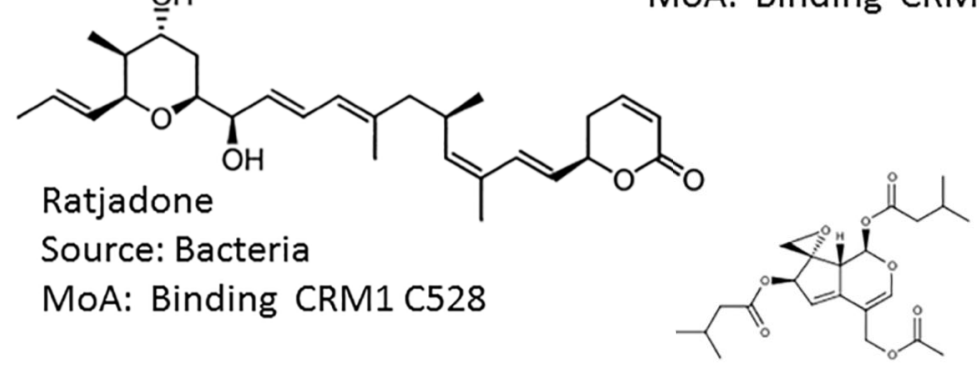

Valtrate

Source: Plant

(1)

Anguinomycin C

Source: Bacteria

MoA: Binding CRM1 C528

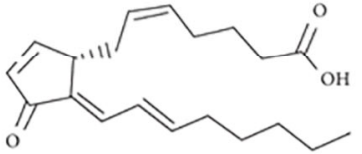

15d-PGJ2

Source: Human

MoA: Binding CRM 1 C528 
<smiles>CC1=CC(=O)c2c(O)cccc2C1=O</smiles>

Plumbagin

Source: Plant

MoA: Binding CRM1 C528<smiles>COc1cc(/C=C/C(=O)CC(=O)/C=C/c2ccc(O)c(OC)c2)ccc1O</smiles>

Curcumin

Source: Plant

MoA: Binding CRM1 C528<smiles>COc1cc(/C=C/C(=O)N2CC=CC2=O)cc(OC)c1OC</smiles>

Piperlongumine

Source: Plant

MoA: Binding CRM1 C528

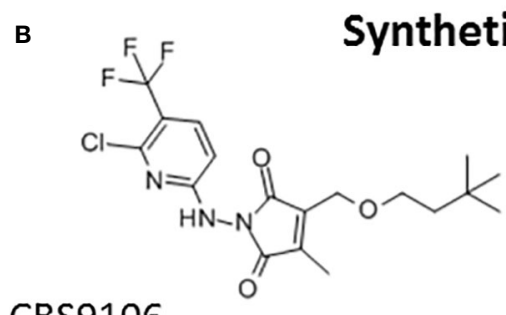

CBS9106

MoA: Binding CRM1 C528<smiles>O=C(/C=C\n1cnc(-c2cc(C(F)(F)F)cc(C(F)(F)F)c2)n1)NNc1cnccn1</smiles>

Selinexor (KPT-330)

MoA: Binding CRM1 C528

FIGURE 2 | Structures of CRM1 inhibitors. (A) Natural compounds: (1) Leptomycin B, (2) Acetoxychavicol acetate (3) Ratjadone, (4) Valtrate, (5) Anguinomycin C, (6) 15d-PGJ2, (7) Plumbagin, (8) Curcumin (9) Piperlongumine (B) Synthetic compounds: (10) CBS9106, (11) KPT-330 (Selinexor).

\section{TARGETING THE CRM1-DRIVEN NUCLEAR EXPORT}

Due to the crucial regulatory role and the alteration in human cancer, CRM1 has emerged as a therapeutic target for anticancer therapy. Although, altered CRM1 expression or activity is not always the driving force behind protein mislocalization, the inhibition of the nuclear export can prevent or correct aberrant subcellular protein localization (Hung and Link, 2011). For example, FOXO proteins are shuttled from the cell nucleus where they can act as tumor suppressors to the cytoplasm via CRM1-mediated nuclear export when they are phosphorylated by the AKT. AKT is a serine/threonine protein kinase and a key component of the PI3K/AKT signaling pathway, which is thought to be the most frequently activated signaling pathway in human cancer. While NEIs do not interfere with the signaling event that led to cytoplasmic mislocalization of FOXOs, they can trap FOXO factors in the cell nucleus and thereby promote their tumor suppressive function. Indeed, the clinically approved NEI Selinexor partially acts through trapping FOXO into the nucleus (Corno et al., 2018). Therefore, NEIs might not only be useful to treat tumors with altered CMR1 expression or function, but relocalize many tumor suppressor proteins or even mislocalize and thereby inactivate oncogenic proteins (Hung and Link, 
2011). Although the groundwork to understand CRM1-mediated nuclear export has been developed over the last decades and the first generation of NEIs including LMB turned out to be to toxic to be used in the clinic, only more recently a significant therapeutic window for these inhibitors has been reported (Mutka et al., 2009). The therapeutic indications of these inhibitors are not limited to cancer but have also the potential to be used as antiviral agents.

\section{Natural Product and Synthetic NEls}

The known NEIs can be classified into natural products and synthetic NEIs (Figures 2A, B). Natural product NEIs are derived from bacterial, plant, fungal or animal sources (Table 1) (Sun et al., 2016). The bacterial NEIs contain a polyketide chain with a lactone ring and include $\mathrm{LMB}$, anguinomycin $\mathrm{A} / \mathrm{B} / \mathrm{C} / \mathrm{D}$ and ratjadone A/C (Hamamoto et al., 1983; Köster et al., 2003; Bonazzi et al., 2010). Anguinomycins are analogs of LMB isolated from Streptomyces sp. Ratjadone is a cytotoxin isolated from myxobacteria from soil at Cala Ratjada on Mallorca island. These polyketide natural products covalently bind to Cys-528 in the human CRM1 and have IC50 values in the low nanomolar range (Sun et al., 2013). However, these NEIs are associated with severe dose limiting toxicities. While they are very powerful tools to study CRM1 function, they are not useful as therapeutic agents. NEIs derived from plants include acetoxychavicol acetate, valtrate, piperlongumine, curcumin, dibenzylideneacetone, gonionthalamin, and plumbagin. They are thought to bind to Cys528 of CRM1 with low affinity and inhibit CRM1 in the micromolar range. Acetoxychavicol acetate (ACA) is found in Alpinia galangal and was identified as an inhibitor of CRM1dependent nuclear export by screening extracts from more than 600 medical plants (Ye and Li, 2006). Pharmacophore features of ACA have been defined. Similar to ACA, the chemically unrelated valtrate was found through screening 200 plant extracts. Valtrate is an iridoid ester with moderate lipophilicity extracted from Valerianae radix (Murakami et al., 2002). Both, ACA and valtrate have been shown to inhibit virus production (Watanabe et al., 2011). Furthermore, two food additives, namely piperlongumine and curcumin were found to inhibit CRM1mediated nuclear export. The alkaloid piperlongumine is extracted from Piper longum Linn and exhibits multiple beneficial effects including selective cytotoxicity against several cancer cell lines. Accordingly, piperlongumine was shown to inhibit the CRM1 dependent transport of tumor suppressor proteins including FOXO1 and p21 (Niu et al., 2015b). Curcumin is a polyphenol compound present in Curcuma longa plant and is the main component of the Indian spice turmeric. It is known for its antioxidant, anti-cancer and antiinflammatory effects. Curcumin and its structural analogue dibenzylideneacetone were shown to prevent the cytoplasmic accumulation of a reporter protein fused with NES in a CRM1dependent manner (Niu et al., 2013). Similarly, a nuclear export assay and molecular modeling were used to characterize the styryl-lactone compound goniothalamin present in Goniothalamus macrophyllus as an inhibitor of nucleocytoplasmic transport via CRM1 (Wach et al., 2010). The natural bicyclic naphthoquinone plumbagin found in roots of plumbaginaceae which have been used in Indian traditional medicine was also identified as a CRM1 inhibitor and capable of blocking the nuclear export of RanBP1 and FOXO1 (Liu et al., 2014). Interestingly, the endogenous, antiinflammatory prostaglandin 15d-PGJ2 has been shown to inhibit CRM1 by a LMB-like mechanism but with much less potency (Hilliard et al., 2010). Conversely, the mechanism of action for the diterpenoid oridonin present in Rabdosia rubescens remains to be determined. Oridonin inhibits inflammation and carcinogenesis and has been used in Chinese traditional medicine. It was shown to induce nuclear translocation of Crm1 and to increase the expression and nuclear accumulation of nucleoporin98, involved in Crm1-mediated nuclear export ( $\mathrm{Li}$ et al., 2014).

The failure of the first generation of NEIs in clinical trials raised skepticism about the therapeutic potential of inhibiting nuclear export. Furthermore, preclinical evaluation of the semisynthetic LMB derivative KOS-2462 (Mutka et al., 2009) and the synthetic small molecule CRM1 inhibitor CBS9106 (Sakakibara et al., 2011) did not lead to their clinical development (Parikh et al., 2014). The realization that there is the possibility of targeting nuclear export with an acceptable level of toxicity have spurred the development of second generation, synthetic CRM1 inhibitors, including CBS9106 (Sakakibara et al., 2011), PKF050-638 (Daelemans et al., 2002), 5219668 (Kau et al., 2003), compound3/4 (Monovich et al., 2009) and S109 (Niu et al., 2015a) and Selective Inhibitors of Nuclear Export (SINEs) (Kalid

TABLE 1 | Natural product nuclear export inhibitors.

\begin{tabular}{|c|c|c|c|}
\hline Natural Product & Source & Mode of Action & Effect \\
\hline Leptomycin B & Streptomyces & CRM1-Cys528 & Potent nuclear export inhibition \\
\hline Anguinomycin & Streptomyces & CRM1-Cys528 & Potent nuclear export inhibition \\
\hline Ratjadone & Myxobacteria & CRM1-Cys528 & Potent nuclear export inhibition \\
\hline Acetoxychavicol acetate & Alpinia galangal & CRM1-Cys528 & Inhibit viral production \\
\hline Valtrate & Valerianae Radix & CRM1-Cys528 & Inhibit viral production \\
\hline Piperlongumine & Piper longum & CRM1-Cys528 & Nuclear tumor suppressor proteins \\
\hline Curcumin & Curcuma longa & CRM1-Cys528 & Nuclear reporter protein \\
\hline Gonionthalamin & Goniothalamus macrophyllus & CRM1-Cys528 & Nuclear reporter protein \\
\hline Plumbagin & Plumbaginaceae & CRM1-Cys528 & Nuclear RanBP1 and FOXO1 \\
\hline 15d-PGJ2 & Human prostaglandine & CRM1-Cys528 & Nuclear TFIIA $\alpha$ \\
\hline Oridonin & Rabdosia rubescens & $n / d$ & Increased and nuclear Nup98 \\
\hline MDN-0105 & Fungal metabolite & CRM1 n/d & Nuclear FOXO3, Rev, NF-кB \\
\hline
\end{tabular}


et al., 2012; Lapalombella et al., 2012; Ranganathan et al., 2012). The first molecule of this family of small molecule compounds was found by a screening effort aimed to identify new anti-HIV Rev inhibitors (Daelemans et al., 2002). The authors analyzed a collection of small molecules using a Rev-dependent luciferase reporter assay and identified PKF050-638 as an anti-viral agent. The SINE compounds include selinexor (KPT-330), verdinexor (KPT-335), KPT-185, KPT-276, and KPT-251. SINEs covalently bind to Cysteine 528 residue in a slowly reversible fashion. While a homozygous or heterozygous mutation of Cys528 conferred resistance to SINE treatment, the E571K mutation did not affect their inhibitory efficacy on the CRM1-mediated nuclear export (García-Santisteban et al., 2016; Jardin et al., 2016). Although, LMB and SINE share the same target amino acid residue of CRM1, SINEs are smaller and occupy less space of the NES groove (Lapalombella et al., 2012; Etchin et al., 2013; Haines et al., 2015; Hing et al., 2016). In addition, SINEs don't undergo hydrolysis upon binding to CRM1 and don't form a salt bridge with CRM1 (Etchin et al., 2013; Sun et al., 2013; Haines et al., 2015). Therefore, the binding of SINEs to CRM1 is slowly reversible (Sun et al., 2013). As a consequence inhibition of CRM1 mediated nuclear export is transient which might explain the reduced toxicity of SINEs compared to the one associated with LMB. SINE compounds have been analyzed in preclinical and clinical studies for numerous solid and hematologic cancers. These clinical trials include the treatment of lymphomas (nonHodgkin's and diffuse large B-cell lymphoma), gliomas, sarcomas, breast cancer, lung cancer, pancreatic cancer, Myelodysplastic Syndromes (MDS), acute myeloid leukemia (AML), Acute lymphocytic leukemia (ALL) multiple myeloma, gastric cancer, esophageal cancer, colorectal cancer, prostate cancer, melanoma, thymic cancer, and gynecologic cancers (Wang and Liu, 2019). The spectrum of malignancies evaluated in these clinical trials underlines the broad applicability of CRM1 inhibitors (Wang and Liu, 2019). The recent clinical approval of Selinexor (Xpovio) also known as KPT-330 is the proof of concept for the therapeutic utility of manipulating the nuclear export. Selinexor has been approved for the treatment of patients with relapsed refractory multiple myeloma (Chari et al., 2019). Side effects associated with SINE treatment are less severe as expected from agents capable of inhibiting a core physiological process as the nuclear export of macromolecule.

\section{NATURAL PRODUCTS AS A SOURCE OF NOVEL CRM1 INHIBITORS}

Natural products are considered as an extremely valuable source for the discovery of new drugs against diverse pathologies. As yet only a fraction of the diversity of bioactive compounds has been explored and opportunities for discovering new natural products leading to new drugs are huge. Several approaches for identifying CRM1 Inhibitors have been reported (Box 1). Bacterial CRM1 inhibitors like LMB might provide Streptomyces with the capacity to kill fungi which restrict their growth. CRM1 is essential for the fungus. A change from Cys529 to Ser in a CRM1 mutant of Saccharomyces pombe render the fungus resistant to $\mathrm{LMB}$, whereas Saccharomyces cerevisiae which does not carry a cysteine at that position is LMB insensitive (Kudo et al., 1999b; Yashiroda and Yoshida, 2003). Many organisms might have developed similar or slightly different capacities to fight and resist competitors. Therefore, is not farefetched to expect metabolites from natural origin to represent a rich source of potential nuclear export inhibitors with novel modes of action. Mutating Cys528 is an obvious yet not evaluated possibility of cancer cells to escape the selective pressure imposed by the treatment with covalent CRM1 inhibitors. It is also possible, that the therapeutic efficacy of CRM1 inhibition might also be limited in cancer cells that strongly overexpress CRM1, but increasing the dosing of covalent CRM1 inhibitors is impossible due to on-target and off-target toxicity. For the time being, all known CRM1

\section{BOX 1 | Approaches for identifying CRM1 Inhibitors.}

\section{Approaches for identifying CRM1 Inhibitors}

Several methods are useful in identifying CRM1 inhibitors including gene reporter assays and image-based high content screening.

\section{Gene reporter assay}

Daelemans et al. reported a screening campaign to identify inhibitors of the CRM1-mediated nuclear export of HIV-1 Rev protein using Rev-dependent luciferase reporter gene assay in Jurkat T cells (Daelemans et al., 2002). The reporter gene is flanked by splice sites under the control of the HIV-1 Rev response element (RRE). The cells are co-transfected firefly luciferase reporter gene fused to the 117 gag sequences and the RRE, flanked by the HIV-1 major splice sites, and driven by the CMV promoter and a vector that expresses HIV-1 Rev protein. Inhibitors of the Rev function cause a dose-dependent inhibition of Rev-dependent luciferase expression. The specificity of the compounds on Rev function can be tested on a Rev independent luciferase gene.

\section{High Content Screening (HCS)}

Image-based HCS (Zanella et al., 2010) has been widely used to screen for CRM1 inhibitors. The U2nesRELOC system uses human U2OS osteosarcoma cells that stably express a green fluorescent protein (GFP)-labeled Rev reporter protein that contains a heterologous nuclear export signal (NES). The fluorescent signal of U2nesRELOC cells is exclusively expressed in the cytoplasm. Treatment with NEls such as LMB the fluorescent reporter rapidly accumulates in the nucleus (Cautain et al., 2015a). Acquisition and analysis of images is based on high content screening technology (Zanella et al., 2010). LMB is used as a positive control. As the cell nucleus is stained with 4,6diamidino-2-phenylindole (DAPI), the cell nucleus can be visualized and the definition of its boundaries can support automated image analysis (Machado et al., 2019). In order to identify small molecule agents that inhibit the nuclear export of tumor suppressors from the nucleus via CRM1, we used the U2nesRELOC system to screen a library of natural product extracts from microbial origin. The collection of products was enriched in secondary metabolites (Cautain et al., 2014). 14,000 different extracts were evaluated for the capacity to accumulate the fluorescent signal in the nucleus of the reporter cells. From the 14,000 extracts, 6000 were obtained from fungi, another 6000 were derived from actinomycetes, and the remaining 2000 extracts were of marine actinomycete origin. 12 extracts with nuclear export inhibitory activities that were not associated with previously known active metabolites were identified. After purification of active compounds, several chemical structures of novel nuclear export inhibitors were identified including the fungal metabolite MDN-0105. 
inhibitors act through covalent binding to Cys528 of CRM1. In addition, they all contain a Michael acceptor which makes them prone to target off effects by non-specific interaction with other targets. The perspective to identify and develop non-covalent inhibitors of CRM1 holds promise to increase efficacy by reducing drug resistance and on-target and off-target toxicity.

Natural product drug discovery is associated with specific limitations including the complexity of natural product chemistry, intellectual property landscape and sustainable supply. Bioavailability challenges associated with natural products could potentially be addressed by designing suitable drug delivery strategies.

\section{CONCLUDING REMARKS}

Interrupting the nuclear export of viral and cellular proteins mediated by CRM1 has emerged as an extremely promising therapeutic strategy to treat patients with viral infections and cancer. The clinical approval of the first-in-class CRM1 inhibitor Selinexor has proven the therapeutic potential of CRM1 inhibition for the treatment of cancer. Compounds derived from natural sources hold promise to support the discovery of new NEIs with a novel mode of action. Image-based high content screening of extract and compound collections is a very efficient way to identify natural products with inhibitory effect on the nuclear export.

\section{REFERENCES}

Bonazzi, S., Eidam, O., Güttinger, S., Wach, J. Y., Zemp, I., Kutay, U., et al. (2010). Anguinomycins and derivatives: Total syntheses, modeling, and biological evaluation of the inhibition of nucleocytoplasmic transport. J. Am. Chem. Soc 132, 1432-1442. doi: 10.1021/ja9097093

Cautain, B., de Pedro, N., Murillo Garzon, V., Munoz de Escalona, M., Gonzalez Menendez, V., Tormo, J. R., et al. (2014). High-content screening of natural products reveals novel nuclear export inhibitors. J. Biomol. Screen 19, 57-65. doi: $10.1177 / 1087057113501389$

Cautain, B., de Pedro, N., Reyes, F., and Link, W. (2015a). Image-based identification of nuclear export inhibitors from natural products. Methods Mol. Biol. 1270, 307-319. doi: 10.1007/978-1-4939-2309-0_22

Cautain, B., Hill, R., de Pedro, N., and Link, W. (2015b). Components and regulation of nuclear transport processes. FEBS J. 282, 445-462. doi: 10.1111/ febs. 13163

Chari, A., Vogl, D. T., Gavriatopoulou, M., Nooka, A. K., Yee, A. J., Huff, C. A., et al. (2019). Oral Selinexor-Dexamethasone for Triple-Class Refractory Multiple Myeloma. N. Engl. J. Med. 381, 727-738. doi: 10.1056/nejmoa1903455

Corno, C., Stucchi, S., De Cesare, M., Carenini, N., Stamatakos, S., Ciusani, E., et al. (2018). FoxO-1 contributes to the efficacy of the combination of the XPO1 inhibitor selinexor and cisplatin in ovarian carcinoma preclinical models. Biochem. Pharmacol. 147, 93-103. doi: 10.1016/J.BCP.2017.11.009

Daelemans, D., Afonina, E., Nilsson, J., Werner, G., Kjems, J., De Clercq, E., et al. (2002). A synthetic HIV-1 Rev inhibitor interfering with the CRM1-mediated nuclear export. Proc. Natl. Acad. Sci. U. S. A. 99, 14440-14445. doi: 10.1073/ pnas.212285299

Dian, C., Bernaudat, F., Langer, K., Oliva, M. F., Fornerod, M., Schoehn, G., et al. (2013). Structure of a truncation mutant of the nuclear export factor CRM1 provides insights into the auto-inhibitory role of its C-terminal helix. Structure 21, 1338-1349. doi: 10.1016/j.str.2013.06.003

Dong, X., Biswas, A., and Chook, Y. M. (2009a). Structural basis for assembly and disassembly of the CRM1 nuclear export complex. Nat. Struct. Mol. Biol. 16, 558-560. doi: $10.1038 /$ nsmb.1586

\section{AUTHOR CONTRIBUTIONS}

All authors contributed to the critical discussion, text and figure preparation, and proofreading of the current manuscript.

\section{FUNDING}

This work was supported by Fundação para a Ciência e a Tecnologia (FCT) Research Center Grant UID/BIM/04773/ 2013 Centre for Biomedical Research 1334 and by the Spanish Ministry of Science, Innovation and Universities through Grant RTI2018-094629-B-I00 to WL. BF was supported by FCT-SFRH/ BPD/100434/2014 and Marie Curie Individual Fellowship project TRIBBLES (\#748585). This work was also supported by two LPCC-NRS/Terry Fox grants (2016/2017; 2017/2018).

\section{ACKNOWLEDGMENTS}

The authors would like to acknowledge the contribution of the COST Action CM1407 "Challenging organic syntheses inspired by nature-from natural products chemistry to drug discovery" and COST Action CA17104 "New diagnostic and therapeutic tools against multidrug resistant tumors".

Dong, X., Biswas, A., Süel, K. E., Jackson, L. K., Martinez, R., Gu, H., et al. (2009b). Structural basis for leucine-rich nuclear export signal recognition by CRM1. Nature 458, 1136-1141. doi: 10.1038/nature07975

Etchin, J., Sun, Q., Kentsis, A., Farmer, A., Zhang, Z. C., Sanda, T., et al. (2013). Antileukemic activity of nuclear export inhibitors that spare normal hematopoietic cells. Leukemia 27, 66-74. doi: 10.1038/leu.2012.219

Fornerod, M., Ohno, M., Yoshida, M., and Mattaj, I. W. (1997). CRM1 is an export receptor for leucine-rich nuclear export signals. Cell 90, 1051-1060. doi: 10.1016/S0092-8674(00)80371-2

Fukuda, M., Asano, S., Nakamura, T., Adachi, M., Yoshida, M., Yanagida, M., et al. (1997). CRM1 is responsible for intracellular transport mediated by the nuclear export signal. Nature 390, 308-311. doi: 10.1038/36894

Güttler, T., Madl, T., Neumann, P., Deichsel, D., Corsini, L., Monecke, T., et al. (2010). NES consensus redefined by structures of PKI-type and Rev-type nuclear export signals bound to CRM1. Nat. Struct. Mol. Biol. 17, 1367-1376. doi: 10.1038/nsmb.1931

Gao, W., Lu, C., Chen, L., and Keohavong, P. (2015). Overexpression of CRM1: A characteristic feature in a transformed phenotype of lung carcinogenesis and a molecular target for lung cancer adjuvant therapy. J. Thorac. Oncol. 10, 815825. doi: 10.1097/JTO.0000000000000485

García-Santisteban, I., Arregi, I., Alonso-Mariño, M., Urbaneja, M. A., GarciaVallejo, J. J., Bañuelos, S., et al. (2016). A cellular reporter to evaluate CRM1 nuclear export activity: functional analysis of the cancer-related mutant E571K. Cell. Mol. Life Sci. 73, 4685-4699. doi: 10.1007/s00018-016-2292-0

Haines, J. D., Herbin, O., De La Hera, B., Vidaurre, O. G., Moy, G. A., Sun, Q., et al. (2015). Nuclear export inhibitors avert progression in preclinical models of inflammatory demyelination. Nat. Neurosci. 18, 511-520. doi: 10.1038/nn.3953

Hamamoto, T., GUNJI, S., TSUJI, H., and BEPPU, T. (1983). Leptomycins A and $\mathrm{B}$, new antifungal antibiotics. I. Taxonomy of the producing strain and their fermentation, purification and characterization. J. Antibiot. (Tokyo) 36, 639645. doi: 10.7164/antibiotics.36.639

Hill, R., Cautain, B., de Pedro, N., and Link, W. (2014). Targeting nucleocytoplasmic transport in cancer therapy. Oncotarget 5, 11-28. doi: 10.18632/oncotarget.1457 
Hilliard, M., Frohnert, C., Spillner, C., Marcone, S., Nath, A., Lampe, T., et al. (2010). The anti-inflammatory prostaglandin 15-deoxy- $\Delta 12,14-$ PGJ2 inhibits CRM1-dependent nuclear protein export. J. Biol. Chem. 285, 22202-22210. doi: $10.1074 /$ jbc.M110.131821

Hing, Z. A., Fung, H. Y. J., Ranganathan, P., Mitchell, S., El-Gamal, D., Woyach, J. A., et al. (2016). Next-generation XPO1 inhibitor shows improved efficacy and in vivo tolerability in hematological malignancies. Leukemia 30, 2364-2372. doi: 10.1038/leu.2016.136

Hung, M. C., and Link, W. (2011). Protein localization in disease and therapy. J. Cell Sci. 124, 3381-3392. doi: 10.1242/jcs.089110

Inoue, H., Kauffman, M., Shacham, S., Landesman, Y., Yang, J., Evans, C. P., et al. (2013). CRM1 blockade by selective inhibitors of nuclear export attenuates kidney cancer growth. J. Urol. 189, 2317-2326. doi: 10.1016/j.juro.2012.10.018

Jardin, F., Pujals, A., Pelletier, L., Bohers, E., Camus, V., Mareschal, S., et al. (2016). Recurrent mutations of the exportin 1 gene (XPO1) and their impact on selective inhibitor of nuclear export compounds sensitivity in primary mediastinal B-cell lymphoma. Am. J. Hematol. 91, 923-930. doi: 10.1002/ ajh. 24451

Köster, M., Lykke-Andersen, S., Elnakady, Y. A., Gerth, K., Washausen, P., Höfle, G., et al. (2003). Ratjadones inhibit nuclear export by blocking CRM1/exportin 1. Exp. Cell Res. 286, 321-331. doi: 10.1016/S0014-4827(03)00100-9

Kalid, O., Toledo Warshaviak, D., Shechter, S., Sherman, W., and Shacham, S. (2012). Consensus Induced Fit Docking (cIFD): methodology, validation, and application to the discovery of novel Crm1 inhibitors. J. Comput. Aided. Mol. Des. 26, 1217-1228. doi: 10.1007/s10822-012-9611-9

Kau, T. R., Schroeder, F., Ramaswamy, S., Wojciechowski, C. L., Zhao, J. J., Roberts, T. M., et al. (2003). A chemical genetic screen identifies inhibitors of regulated nuclear export of a Forkhead transcription factor in PTEN-deficient tumor cells. Cancer Cell 4, 463-476. doi: 10.1016/S1535-6108(03)00303-9

Kojima, K., Kornblau, S. M., Ruvolo, V., Dilip, A., Duvvuri, S., Davis, R. E., et al. (2013). Prognostic impact and targeting of CRM1 in acute myeloid leukemia. Blood 121, 4166-4174. doi: 10.1182/blood-2012-08-447581

Koyama, M., and Matsuura, Y. (2010). An allosteric mechanism to displace nuclear export cargo from CRM1 and RanGTP by RanBP1. EMBO J. 29, 2002-2013. doi: 10.1038/emboj.2010.89

Kudo, N., Wolff, B., Sekimoto, T., Schreiner, E. P., Yoneda, Y., Yanagida, M., et al. (1998). Leptomycin B Inhibition of Signal-Mediated Nuclear Export by Direct Binding to CRM1. Exp. Cell Res. 242, 540-547. doi: 10.1006/excr.1998.4136

Kudo, N., Matsumori, N., Taoka, H., Fujiwara, D., Schreiner, E. P., Wolff, B., et al. (1999a). Leptomycin B inactivates CRM1/exportin 1 by covalent modification at a cysteine residue in the central conserved region. Proc. Natl. Acad. Sci. U. S. A. 96, 9112-9117. doi: 10.1073/pnas.96.16.9112

Kudo, N., Matsumori, N., Taoka, H., Fujiwara, D., Schreiner, E. P., Wolff, B., et al. (1999b). Leptomycin B inactivates CRM1/exportin 1 by covalent modification at a cysteine residue in the central conserved region. Proc. Natl. Acad. Sci. U. S. A. 96, 9112-9117. doi: 10.1073/pnas.96.16.9112

Lapalombella, R., Sun, Q., Williams, K., Tangeman, L., Jha, S., Zhong, Y., et al. (2012). Selective inhibitors of nuclear export show that CRM1/XPO1 is a target in chronic lymphocytic leukemia. Blood 120, 4621-4634. doi: 10.1182/blood2012-05-429506

Li, F. F., Yi, S., Wen, L., He, J., Yang, L. J., Zhao, J., et al. (2014). Oridonin induces NPM mutant protein translocation and apoptosis in NPM1c+ acute myeloid leukemia cells in vitro. Acta Pharmacol. Sin. 35, 806-813. doi: 10.1038/ aps. 2014.25

Liu, X., Niu, M., Xu, X., Cai, W., Zeng, L., Zhou, X., et al. (2014). CRM1 is a direct cellular target of the natural anti-cancer agent plumbagin. J. Pharmacol. Sci. 124, 486-493. doi: 10.1254/jphs.13240FP

Machado, S., Raposo, C., Ferreira, B. I., and Link, W. (2019). Image-based Identification of Chemical Compounds Capable of Trapping FOXO in the Cell Nucleus. Methods Mol. Biol. 1890, 163-170. doi: 10.1007/978-1-49398900-3_14

Monecke, T., Güttler, T., Neumann, P., Dickmanns, A., Görlich, D., and Ficner, R. (2009). Crystal structure of the nuclear export receptor CRM1 in complex with snurportin1 and RanGTP. Sci. (80-.). 324, 1087-1091. doi: 10.1126/ science. 1173388
Monecke, T., Haselbach, D., Voß, B., Russek, A., Neumann, P., Thomson, E., et al. (2013). Structural basis for cooperativity of CRM1 export complex formation. Proc. Natl. Acad. Sci. U. S. A. 110, 960-965. doi: 10.1073/pnas.1215214110

Monovich, L., Koch, K. A., Burgis, R., Osimboni, E., Mann, T., Wall, D., et al. (2009). Suppression of HDAC nuclear export and cardiomyocyte hypertrophy by novel irreversible inhibitors of CRM1. Biochim. Biophys. Acta Gene Regul. Mech. 1789, 422-431. doi: 10.1016/j.bbagrm.2009.04.001

Murakami, N., Ye, Y., Kawanishi, M., Aoki, S., Kudo, N., Yoshida, M., et al. (2002). New Rev-transport inhibitor with anti-HIV activity from Valerianae Radix. Bioorg. Med. Chem. Lett. 12, 2807-2810. doi: 10.1016/S0960-894X(02)00624-8

Mutka, S. C., Yang, W. Q., Dong, S. D., Ward, S. L., Craig, D. A., Timmermans, P. B. M. W. M., et al. (2009). Identification of nuclear export inhibitors with potent anticancer activity in vivo. Cancer Res. 69, 510-517. doi: 10.1158/00085472.CAN-08-0858

Newlands, E. S., Rustin, G. J., and Brampton, M. H. (1996). Phase I trial of elactocin. Br. J. Cancer 74, 648-649. doi: 10.1038/bjc.1996.415

Niu, M., Wu, S., Mao, L., and Yang, Y. (2013). CRM1 Is a Cellular Target of Curcumin: New Insights for the Myriad of Biological Effects of an Ancient Spice. Traffic 14, 1042-1052. doi: 10.1111/tra.12090

Niu, M., Chong, Y., Han, Y., and Liu, X. (2015a). Novel reversible selective inhibitor of nuclear export shows that CRM1 is a target in colorectal cancer cells. Cancer Biol. Ther. 16, 1110-1118. doi: 10.1080/15384047.2015.1047569

Niu, M., Xu, X., Shen, Y., Yao, Y., Qiao, J., Zhu, F., et al. (2015b). Piperlongumine is a novel nuclear export inhibitor with potent anticancer activity. Chem. Biol. Interact. 237, 66-72. doi: 10.1016/j.cbi.2015.05.016

Noske, A., Weichert, W., Niesporek, S., Röske, A., Buckendahl, A.-C., Koch, I., et al. (2008). Expression of the nuclear export protein chromosomal region maintenance/exportin 1/Xpol is a prognostic factor in human ovarian cancer. Cancer 112, 1733-1743. doi: 10.1002/cncr.23354

Ossareh-Nazari, B., Bachelerie, F., and Dargemont, C. (1997). Evidence for a role of CRM1 in signal-mediated nuclear protein export. Sci. (80-. ). 278, 141-144. doi: 10.1126/science.278.5335.141

Parikh, K., Cang, S., Sekhri, A., and Liu, D. (2014). Selective inhibitors of nuclear export (SINE)-A novel class of anti-cancer agents. J. Hematol. Oncol. 7, 78. doi: 10.1186/s13045-014-0078-0

Puente, X. S., Pinyol, M., Quesada, V., Conde, L., Ordóñez, G. R., Villamor, N., et al. (2011). Whole-genome sequencing identifies recurrent mutations in chronic lymphocytic leukaemia. Nature 475, 101-105. doi: 10.1038/ nature 10113

Ranganathan, P., Yu, X., Na, C., Santhanam, R., Shacham, S., Kauffman, M., et al. (2012). Preclinical activity of a novel CRM1 inhibitor in acute myeloid leukemia. Blood 120, 1765-1773. doi: 10.1182/blood-2012-04-423160

Saito, N., and Matsuura, Y. (2013). A 2.1- $\AA$-resolution crystal structure of unliganded CRM1 reveals the mechanism of autoinhibition. J. Mol. Biol. 425, 350-364. doi: 10.1016/j.jmb.2012.11.014

Sakakibara, K., Saito, N., Sato, T., Suzuki, A., Hasegawa, Y., Friedman, J. M., et al. (2011). CBS9106 is a novel reversible oral CRM1 inhibitor with CRM1 degrading activity. Blood 118, 3922-3931. doi: 10.1182/blood-2011-01333138

Santiago, A., Li, D., Zhao, L. Y., Godsey, A., and Liao, D. (2013). P53 SUMOylation promotes its nuclear export by facilitating its release from the nuclear export receptor CRM1. Mol. Biol. Cell 24, 2739-2752. doi: 10.1091/mbc.E12-10-0771

Schmidt, J., Braggio, E., Kortuem, K. M., Egan, J. B., Zhu, Y. X., Xin, C. S., et al. (2013). Genome-wide studies in multiple myeloma identify XPO1/CRM1 as a critical target validated using the selective nuclear export inhibitor KPT-276. Leukemia 27, 2357-2365. doi: 10.1038/leu.2013.172

Sendino, M., Omaetxebarria, M. J., and Rodríguez, J. A. (2018). Hitting a moving target: inhibition of the nuclear export receptor XPO1/CRM1 as a therapeutic approach in cancer. Cancer Drug Resist 1, 139-163. doi: 10.20517/cdr.2018.09

Shen, A., Wang, Y., Zhao, Y., Zou, L., Sun, L., and Cheng, C. (2009). Expression of crm1 in human gliomas and its significance in p27 expression and clinical prognosis. Neurosurgery 65, 153-160. doi: 10.1227/01.NEU.0000348550.47441.4B

Sun, Q., Carrasco, Y. P., Hu, Y., Guo, X., Mirzaei, H., MacMillan, J., et al. (2013). Nuclear export inhibition through covalent conjugation and hydrolysis of 
Leptomycin B by CRM1. Proc. Natl. Acad. Sci. U. S. A. 110, 1303-1308. doi: 10.1073/pnas.1217203110

Sun, Q., Chen, X., Zhou, Q., Burstein, E., Yang, S., and Jia, D. (2016). Inhibiting cancer cell hallmark features through nuclear export inhibition. Signal Transduction Targeting Ther. 1, 16010. doi: 10.1038/sigtrans.2016.10

Tai, Y. T., Landesman, Y., Acharya, C., Calle, Y., Zhong, M. Y., Cea, M., et al. (2014). CRM1 inhibition induces tumor cell cytotoxicity and impairs osteoclastogenesis in multiple myeloma: Molecular mechanisms and therapeutic implications. Leukemia 28, 155-165. doi: 10.1038/ leu.2013.115

Turner, J., and Sullivan, D. (2008). CRM1-Mediated Nuclear Export of Proteins and Drug Resistance in Cancer. Curr. Med. Chem. 15, 2648-2655. doi: 10.2174/ 092986708786242859

Turner, J. G., Dawson, J., and Sullivan, D. M. (2012). Nuclear export of proteins and drug resistance in cancer. Biochem. Pharmacol. 83, 1021-1032. doi: 10.1016/j.bcp.2011.12.016

van der Watt, P. J., Maske, C. P., Hendricks, D. T., Parker, M. I., Denny, L., Govender, D., et al. (2009). The Karyopherin proteins, Crm1 and Karyopherin $\beta 1$, are overexpressed in cervical cancer and are critical for cancer cell survival and proliferation. Int. J. Cancer 124, 1829-1840. doi: 10.1002/ijc.24146

Van Der Watt, P. J., Zemanay, W., Govender, D., Hendricks, D. T., Parker, M. I., and Leaner, V. D. (2014). Elevated expression of the nuclear export protein, Crm1 (exportin 1), associates with human oesophageal squamous cell carcinoma. Oncol. Rep. 32, 730-738. doi: 10.3892/or.2014.3231

Wach, J. Y., Güttinger, S., Kutay, U., and Gademann, K. (2010). The cytotoxic styryl lactone goniothalamin is an inhibitor of nucleocytoplasmic transport. Bioorg. Med. Chem. Lett. 20, 2843-2846. doi: 10.1016/j.bmcl.2010.03.049

Wang, A. Y., and Liu, H. (2019). The past, present, and future of CRM1/XPO1 inhibitors. Stem Cell Investig. 6, 6. doi: 10.21037/sci.2019.02.03

Watanabe, K., Takatsuki, H., Sonoda, M., Tamura, S., Murakami, N., and Kobayashi, N. (2011). Anti-influenza viral effects of novel nuclear export inhibitors from Valerianae Radix and Alpinia galanga. Drug Discovery Ther. 5, 26-31. doi: 10.5582/ddt.v5.1.26
Yao, Y., Dong, Y., Lin, F., Zhao, H., Shen, Z., Chen, P., et al. (2009). The expression of CRM1 is associated with prognosis in human osteosarcoma. Oncol. Rep. 21, 229-235. doi: 10.3892/or_00000213

Yashiroda, Y., and Yoshida, M. (2003). Nucleo-Cytoplasmic Transport of Proteins as a Target for Therapeutic Drugs. Curr. Med. Chem. 10, 741-748. doi: $10.2174 / 0929867033457791$

Ye, Y., and Li, B. (2006). 1'S-1'-acetoxychavicol acetate isolated from Alpinia galanga inhibits human immunodeficiency virus type 1 replication by blocking Rev transport. J. Gen. Virol. 87, 2047-2053. doi: 10.1099/vir.0.81685-0

Yoshimura, M., Ishizawa, J., Ruvolo, V., Dilip, A., Quintás-Cardama, A., McDonnell, T. J., et al. (2014). Induction of p53-mediated transcription and apoptosis by exportin-1 (XPO1) inhibition in mantle cell lymphoma. Cancer Sci. 105, 795-801. doi: $10.1111 /$ cas. 12430

Zanella, F., Lorens, J. B., and Link, W. (2010). High content screening: seeing is believing. Trends Biotechnol. 28, 237-245. doi: 10.1016/j.tibtech.2010.02.005

Zheng, Y., Gery, S., Sun, H., Shacham, S., Kauffman, M., and Koeffler, H. P. (2014). KPT-330 inhibitor of XPO1-mediated nuclear export has anti-proliferative activity in hepatocellular carcinoma. Cancer Chemother. Pharmacol. 74, 487495. doi: 10.1007/s00280-014-2495-8

Zhou, F., Qiu, W., Yao, R., Xiang, J., Sun, X., Liu, S., et al. (2013). CRM1 is a novel independent prognostic factor for the poor prognosis of gastric carcinomas. Med. Oncol. 30 (726). doi: 10.1007/s12032-013-0726-1

Conflict of Interest: The authors declare that the research was conducted in the absence of any commercial or financial relationships that could be construed as a potential conflict of interest.

Copyright $\odot 2020$ Ferreira, Cautain, Grenho and Link. This is an open-access article distributed under the terms of the Creative Commons Attribution License (CC BY). The use, distribution or reproduction in other forums is permitted, provided the original author(s) and the copyright owner(s) are credited and that the original publication in this journal is cited, in accordance with accepted academic practice. No use, distribution or reproduction is permitted which does not comply with these terms. 